\title{
A Comparison of Reliability and Construct Validity between the Original and Revised Versions of the Rosenberg Self-Esteem Scale
}

Wongpakaran Tinakon and Wongpakaran Nahathai

Department of Psychiatry, Faculty of Medicine, Chiang Mai University, Thailand

Psychiatry Investig 2012;9:54-58

http://dx.doi.org/10.4306/pi.2012.9.1.54

The name of the first author should be "Tinakon Wongpakaran" instead of "Wongpakaran Tinakon." The name of the second author should be "Nahathai Wongpakaran" instead of "Wongpakaran Nahathai." The authors sincerely regret this error.

Corrected author's name

Tinakon Wongpakaran and Nahathai Wongpakaran 